\title{
Rheology Control of Thickened Tailings and Paste Pumping Systems
}

\author{
A.J.C. Paterson, Paterson and Cooke Consulting Engineers (Pty) Ltd, South Africa
}

\section{INTRODUCTION}

Thickened and paste tailings typically exhibit non-Newtonian characteristics. It is well established that the viscous properties of these slurries are dependent on a wide range of parameters, including particle size distribution, solids concentration, $\mathrm{pH}$ and water chemistry. However, many systems are still designed assuming a constant rheology, and operating conditions are specified in terms of slurry density. At low solids concentrations this is acceptable as the effects of changes in solids concentration on rheology are relatively small. As solids concentration increases, small increments have a significant impact on rheology. Designing for a constant solids concentration or density implies (unrealistically) that the ore body will be uniform throughout the mine life and results in systems that are not sufficiently flexible to accommodate the wide fluctuations in rheology that will occur.

This paper presents an alternative methodology that assumes that solids concentration will vary naturally as physical properties change, and that rheology is the key driver to ensure a reliable, and integrated, system design. The benefits are numerous, including improved thickener control, optimum pumping system design, and obtaining consistent deposition and beaching angles.

\section{WHAT IS RHEOLOGY?}

Rheology is a term that is loosely applied to many processes, often incorrectly, and the term needs to be properly understood. Rheology is the science of the deformation and flow of matter ${ }^{1}$; the study of the manner in which materials respond to applied stress or strain. In theory all materials have rheological properties that affect their behaviour; from long term creep phenomena measured over eons on a geological scale, to high speed process environments such as thin film coating in the paper industry.

In the mineral processing industry, the rheological properties of the ores affect all aspects of the beneficiation process to some extent. The rheological property that most affects beneficiation is essentially the "viscosity" of the product. Qualitatively, the viscosity is the property of the material to resist deformation increasingly

\footnotetext{
${ }^{1}$ British Standards Institution BS 5168 "Glossary of Rheological Terms".
} 
with increasing rate of deformation. As a quantitative measure, it is defined as the ratio of shear stress divided by shear rate in steady flow, as shown in Equation 1 for Newtonian Fluids:

$$
\text { Shear Stress, } \tau=\mu \gamma
$$

$$
\text { where } \begin{aligned}
& \mu=\operatorname{viscosity}(\mathrm{Pa} . \mathrm{s}) \\
& \tau=\operatorname{shear} \text { stress }(\mathrm{Pa}) \\
& \dot{\gamma}=\operatorname{shear} \operatorname{rate}\left(\mathrm{s}^{-1}\right) .
\end{aligned}
$$

For Newtonian fluids, this relationship is linear and the viscosity completely defines the flow behaviour when the flow is laminar. If the relationship is not linear, then the fluid is non-Newtonian and additional parameters, such as yield stress, dynamic and elastic properties, and flow behaviour index, are required to characterise the fluid. The difference between a Newtonian and non-Newtonian fluid is shown in the rheogram in Figure 1. A rheogram is a graphical representation of the shear stress versus shear stress relationship. For a Newtonian fluid the rheogram is defined by the viscosity, or slope of the rheogram, which is constant for all shear rates. It is seen that for a non-Newtonian fluid, the ratio of shear stress over shear rate varies. This means that the viscosity is not constant and is dependent on the shear rate. The terms "apparent viscosity" or "secant viscosity" are sometimes used to define the viscosity at a given shear rate.

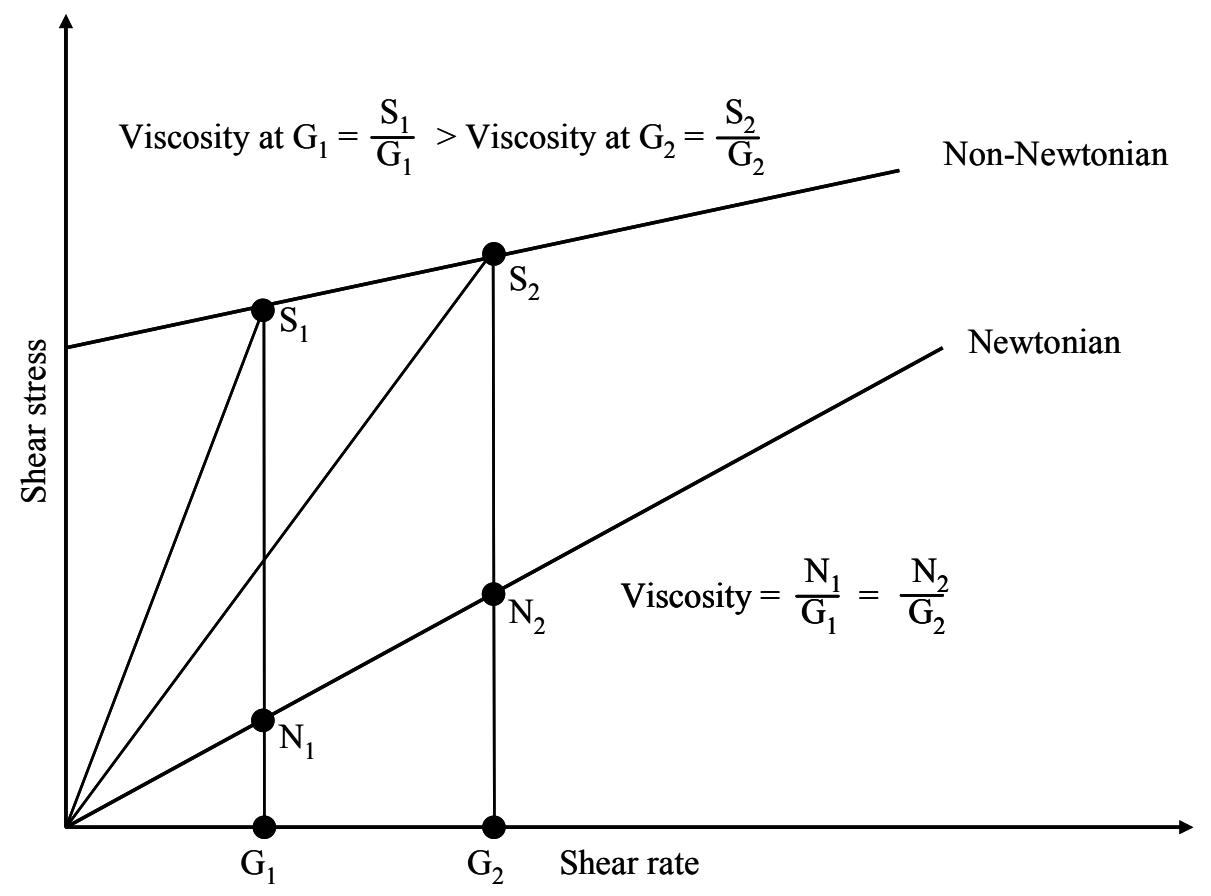

\section{Figure $1 \quad$ Variation of viscosity of Newtonian and non-Newtonian fluids}

The rheogram of the non-Newtonian fluid shown in Figure 1 is for a time independent fluid, which means that the relationship does not vary as a function of the shear history or duration of shear. Time dependent slurries are those for which the shear stress is dependent upon both shear rate and duration of applied shear. 
This means that the rheogram is not unique and depends on the history of the shear rate. This paper discusses time independent fluids that commonly occur in mineral suspensions. Typical time independent fluids are Bingham Plastic, pseudo plastic, or yield pseudo plastic fluids. The Bingham Plastic model is the most widely used approximation to the rheogram for mineral suspensions and is characterised by two parameters; the Bingham yield stress and the plastic viscosity, as shown in Equation 2. Note that several definitions for yield stress exist, the most simple being the point at which the rheogram meets the stress axis. For practical purposes, this is the stress required to initiate measurable flow.

$$
\tau=\tau_{\mathrm{y}}+\eta_{\mathrm{p}} \gamma
$$

where $\tau_{\mathrm{y}}=$ Bingham yield stress $(\mathrm{Pa})$

$\eta_{\mathrm{p}}=$ plastic viscosity (Pa.s).

\subsection{Pipe flow}

Non-Newtonian pipe flow can be either laminar or turbulent. Laminar flow occurs at low Reynolds Numbers when the "viscous" properties of the fluid dominate, and turbulent flow occurs when inertial properties dominate. For Newtonian fluids it is a straightforward matter to determine the Reynolds Number as the viscous properties are constant. For non-Newtonian fluids there are several methods available to estimate whether flow is laminar or turbulent that are beyond the scope of this paper. However, a very useful guideline to determine the change from laminar to turbulent flow is the method proposed by Slatter and Wasp (2002) for Bingham Plastic slurries. A wide range of slurry data were analysed and correlated to different Hedström Numbers. At high Hedström Numbers it was observed that the critical velocity, $\mathrm{V}_{\text {crit, }}$ was independent of pipeline diameter and fluid consistency index, and was largely a function of the yield stress as shown in Equation 3:

$$
\mathrm{V}_{\text {crit }}=26 \sqrt{\frac{\tau_{\mathrm{y}}}{\rho_{\mathrm{m}}}}
$$

where $\rho_{\mathrm{m}}=$ slurry density $\left(\mathrm{kg} / \mathrm{m}^{3}\right)$.

\subsubsection{Typical pressure loss relationship}

A typical pressure loss relationship for a Bingham Plastic fluid is shown in Figure 2 at different slurry densities. As density increases, for a particular pipeline velocity the relative increase in pressure gradient is considerably more when flow is laminar than when it is turbulent. Note that the turbulent flow data lie on approximately the same curve. 


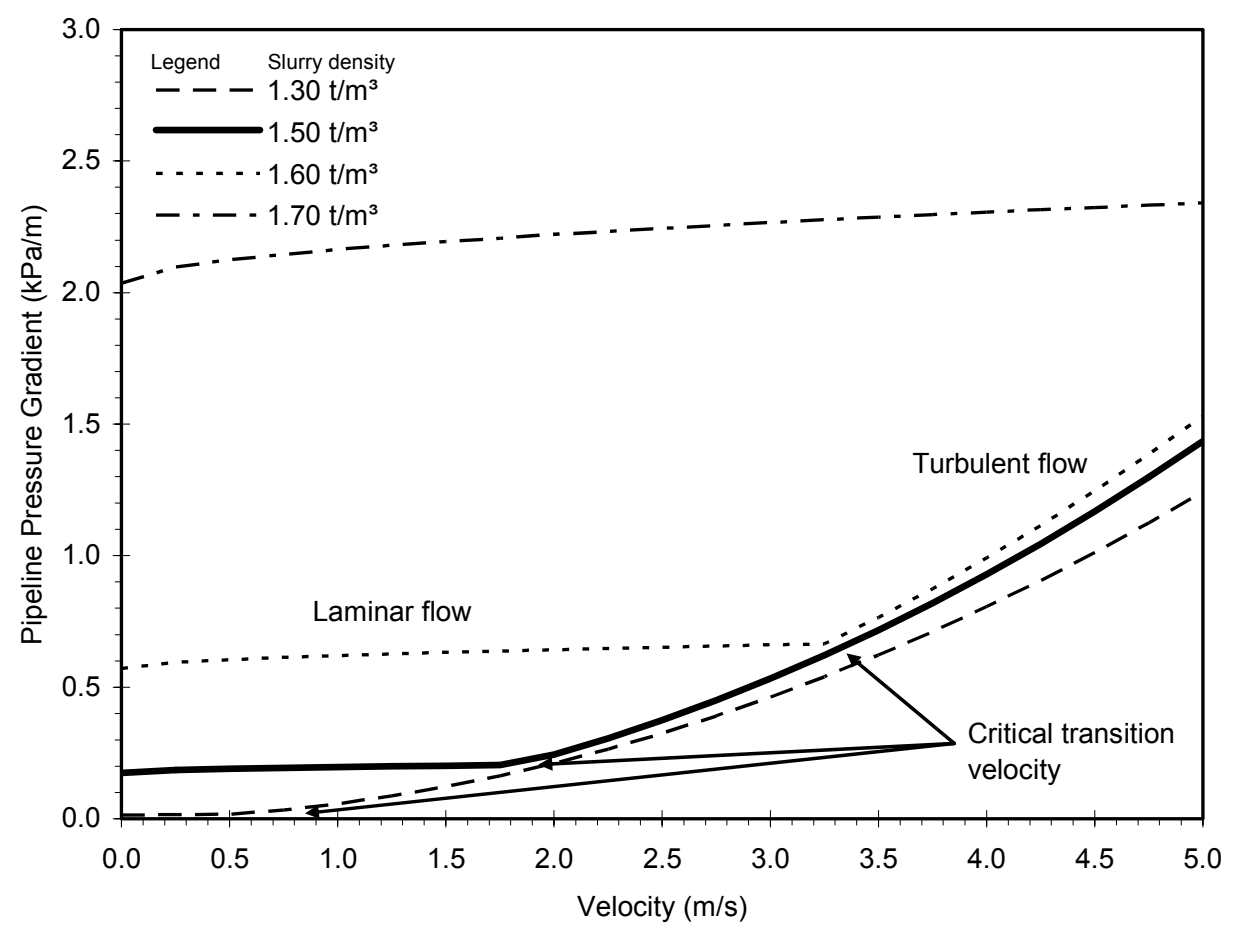

\section{Figure 2 Pressure loss versus velocity as a function of slurry density $(200 \mathrm{~mm}$ pipeline)}

In Figure 2, rheology dominates the laminar flow regime. Note that the rheological properties of a fluid can only be measured when the flow is laminar. At a particular shear rate, or corresponding pipeline velocity, the shear stress or pressure gradient can be determined using the apparent viscosity at that particular shear rate, provided the flow is laminar. If the flow is turbulent, then this cannot be done and the pressure loss needs to be determined using conventional calculation methods.

\section{PARADIGM SHIFT}

\subsection{Density control}

Conventional low-density slurry pumping systems behave in a very different way from high-density thickened tailings systems, and this difference becomes more pronounced as density increases. At low densities the slurry velocity must be sufficiently high to prevent solids depositing on the pipeline invert, and as the viscous properties are insignificant, the flow will likely to be turbulent. From Figure 2, it is clear that any changes in density will have a small effect on the flow properties, and consequently the system performance is not affected. This is true for most conventional systems, as the relationship between rheology and density in this range is not exponential, as is the case at high densities. Rheology is influenced by many parameters other than density, such as particle size and shape, $\mathrm{pH}$, water chemistry and colloidal properties. These are factors that vary as the host ore-body changes and on most mining operations, the properties of the ore varies within the ore-body. Figure 3 shows that the change in viscous properties as a 
function of density at low densities is often not substantial for the same ore. For a given density the yield stress is similar.

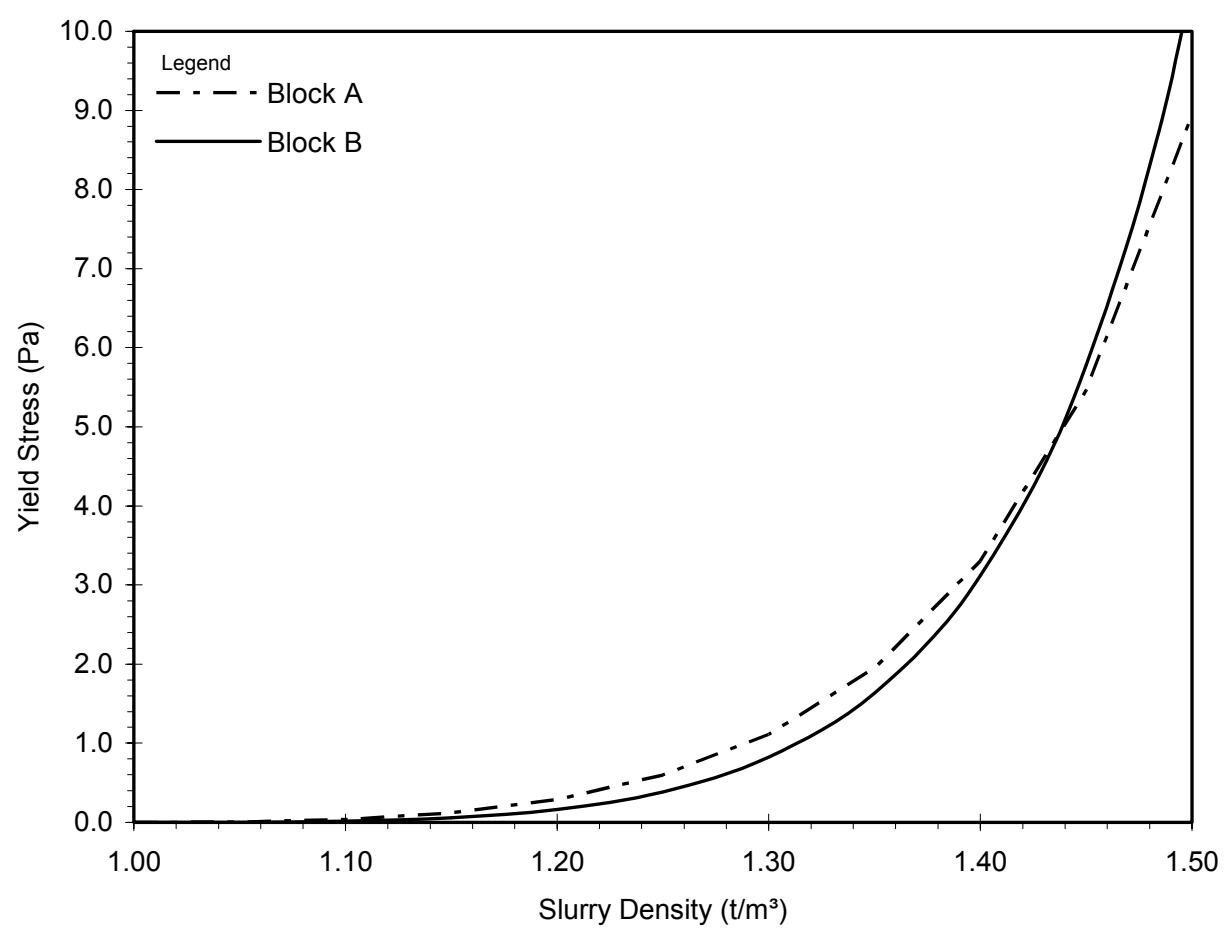

Figure 3 Change in yield stress at low slurry densities

The typical relationship in Figure 3 is the underlying reason why "conventional" disposal systems have historically been controlled on density. Conventional thickeners are controlled on the basis of the underflow density and provided that the slurry is not very viscous, this is a robust and easy to measure control parameter.

\subsection{Rheology control}

The advancement of thickening technology, and the drive to increase solids concentration, has fundamentally changed the requirements for controlling these systems. However, this is often overlooked. As solids density increases, the increase in the viscous properties becomes exponential as can be seen in Figure 4 where at high densities there is a substantial, exponential, change in rheology as density varies. A process that is controlled on density alone will not be able to manage the change in the viscous properties of the different ore types, as at a high density there is a significant difference in the rheology of the different ore types that will affect the beneficiation process.

Figure 4 represents the typical nature of the yield stress versus density or solids concentration. Values will differ as ores changes, but at some point the exponential relationship becomes the dominant feature of the material behaviour. This means that the concept of density control is no longer valid and processes must be controlled according to the viscous properties of the fluid. 


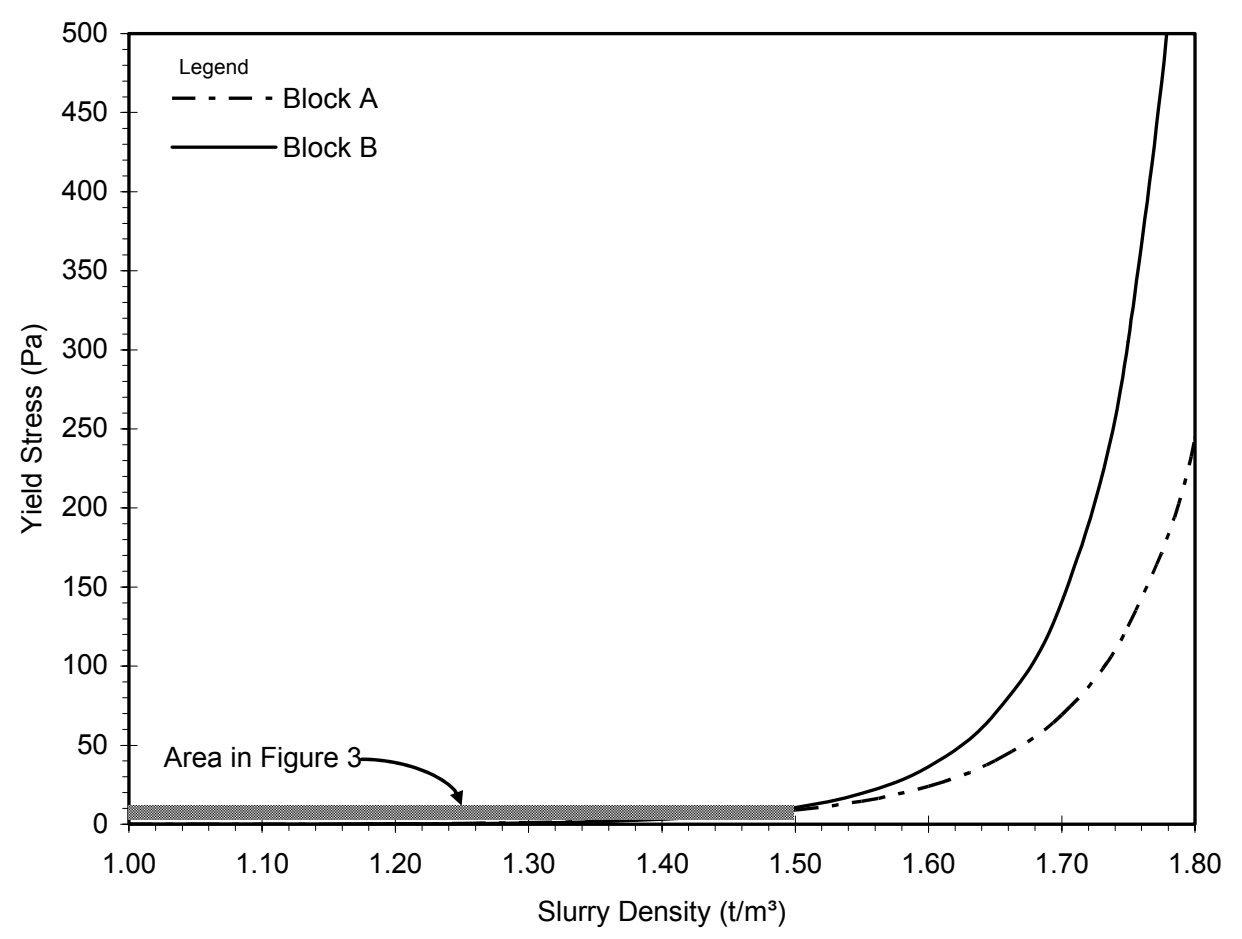

\section{Figure 4 Change in yield stress at high slurry densities}

\section{RHEOLOGY CONTROL}

\subsection{Pipeline systems}

The point at which rheology becomes an important control parameter in a pipeline system can be estimated for a Bingham slurry by using Equation 3 as a guideline. A key parameter for any slurry pipeline system is the selection of the appropriate operating velocity. For practical purposes, to limit pipeline wear and to minimise pressure losses, it is unlikely that a system would be designed to operate at slurry velocities very much greater than about $4 \mathrm{~m} / \mathrm{s}$ (which is already high). Figure 5 shows the relationship between Equation 3 and the yield stress correlations in Figures 3 and 4. When the yield stress is greater than about $40 \mathrm{~Pa}$, the velocity in the pipeline for a Bingham Plastic slurry, will be too high for practical purposes more or less regardless of diameter. In Figure 4, a yield stress of $40 \mathrm{~Pa}$ corresponds to a density of $\pm 1.60 \mathrm{t} / \mathrm{m}^{3}$ for Block B and $1.65 \mathrm{t} / \mathrm{m}^{3}$ for Block A. Above a density of $1.60 \mathrm{t} / \mathrm{m}^{3}$ the system will need to be controlled on the basis of rheology and not the density, as it is seen that from this point onwards the rheology of Block A and Block B diverge considerably. 


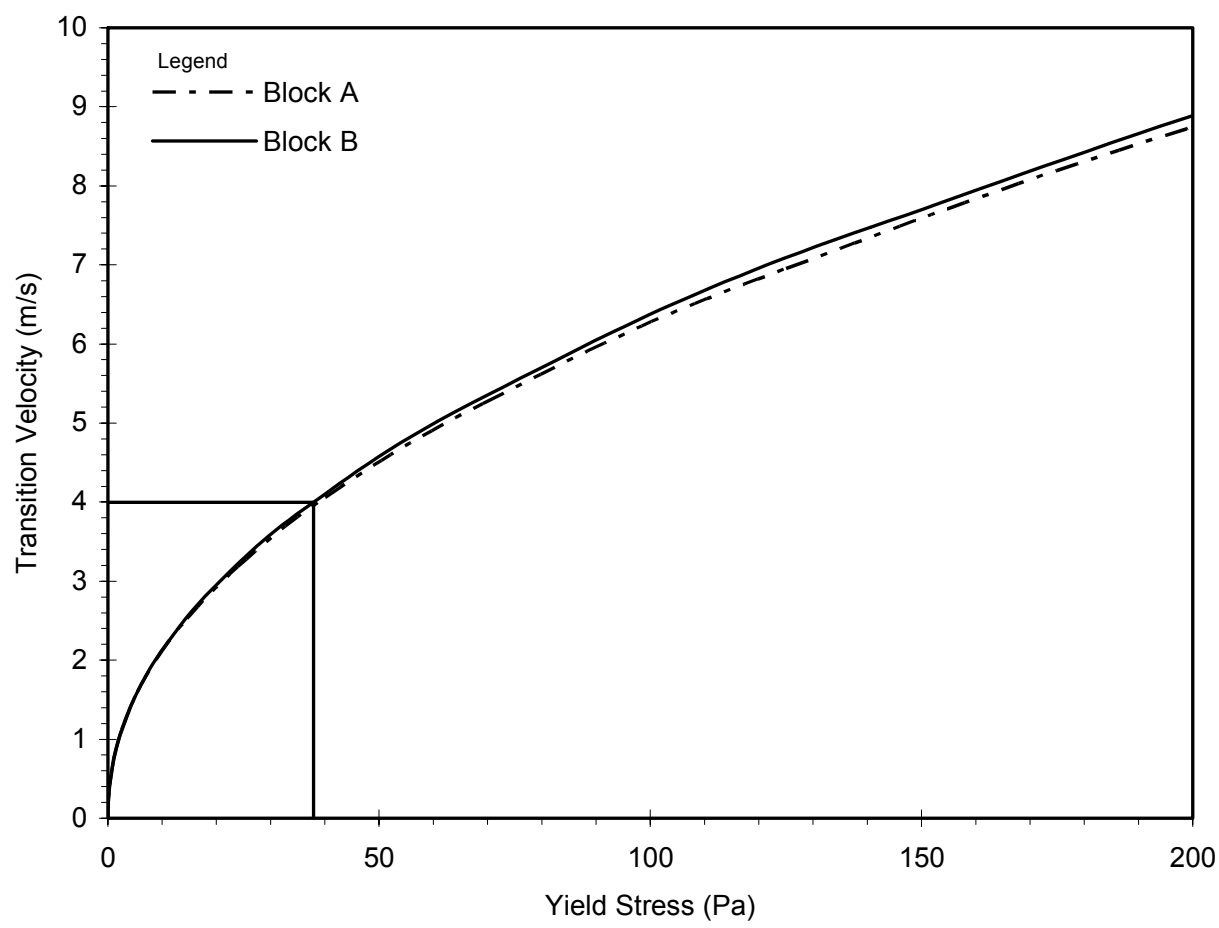

Figure $5 \quad$ Yield stress versus transition velocity

As Figure 5 is independent of pipe diameter, any Bingham Plastic mineral suspension process that has a yield stress greater than approximately $40 \mathrm{~Pa}$ should incorporate a viscosity control system as opposed to a density controlled system. For a pump and pipeline, the system should be designed to operate at the maximum installed pressure capacity, regardless of density. This is undertaken by ensuring the slurry has a consistent rheology. The thickeners or slurry preparation facility must produce consistent rheology and not density.

\subsection{Open channel flow}

Canals or launders are often used to transport thickened underflow to the deposition site, particularly when there is sufficient gravity head available. Open channel flow, as with pipe flow, is very susceptible to the viscous properties of the slurry. Many installations utilising canals to transport thickened tailings have problems using a density based control system, as the canal transport system fails when the ore is more viscous than usual. As viscosity increases, the capacity of a fixed geometry canal is reduced, because as the depth increases the velocity decreases and solids settle on the floor of the canal. Steeper slopes are needed to transport more viscous slurries. Steep slopes are problematic when the viscosity decreases, as the velocity in the open channel increases and the flow becomes unstable, with the potential for hydraulic jumps as the flow regime changes from streaming to shooting flow. As canals are sensitive to the flow regime, it is important to ensure consistent rheology material is available. 


\subsection{Thickener systems}

Thickeners mostly use underflow density as a control parameter, but also consider rake torque. Rake torque is a measure of the rheology of the material in the slurry bed. However, the yield stress of the bed material is often very different from the yield stress recorded in pipelines. One of the reasons is that the thickener bed is partially sheared by the rake pickets, whereas once the material passes the underflow centrifugal pump it is fully sheared by the impeller. The effect of shearing the slurry in the underflow pump can be significant as it breaks down the flocculant structure that may exist in the compressed thickener bed. Generally when a material has passed through a centrifugal pump it is reasonably fully sheared, and this is the material that has to be delivered to the deposition site either via pumps and pipelines, or canals. A thickener that is controlled primarily on density will not be able to compensate for variations in the feed properties, with the result that it may deliver a material with the same density but very different rheology. The consequence is that the transport system may not be able to pump a very viscous material, or if the viscosity is too low, then there is excess pumping capacity that is not utilised.

Flocculant consumption is linked to settling rate in the thickener and a density based control system will add additional flocculant to achieve a density that may require excessive flocculant as it is more viscous. Controlling on rheology will also reduce flocculant consumption as the settling properties of the material are linked to the viscous properties.

\subsection{Deposition}

Obtaining consistent beach angles on a thickened tailings deposition site is the key to ensuring maximum utilisation of the site geometry. Beach angles are a function of both density and rheology, and consistent rheology will ensure that beach angles are consistent.

\subsection{Applications}

Figure 6 shows typical applications where rheology can be measured. Multiple thickeners discharging to a central sump or storage tank each deliver consistent rheology material that is either pumped or transported in a canal. If a single thickener discharges to a pump and pipeline with no break tank, then controlling the rheology will ensure that the system will not overpressure when the pipeline length changes. If the material is shear sensitive and the rheology is affected by the duration of shear in the pipeline, then it is possible to measure rheology at the pipeline discharge to ensure that the minimum beach slope requirements are met. 


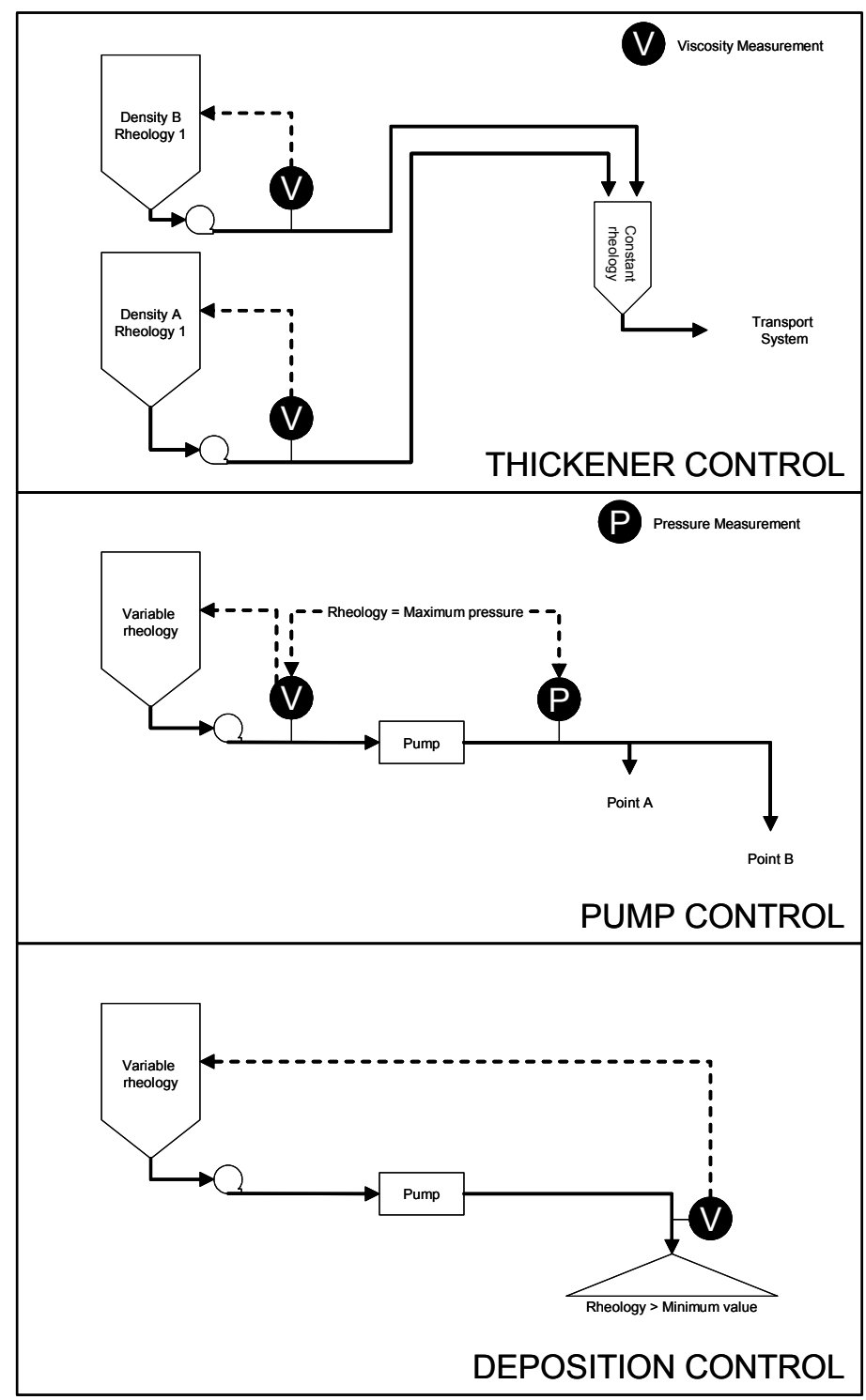

Figure 6 Viscosity control applications

\section{CONCLUSIONS}

An understanding of rheology and its impact on slurry preparation, pumping and deposition aspects has shown that when slurry concentrations are high, density is no longer a suitable control parameter.

As most mineral suspensions can be approximated by the Bingham Plastic model, this paper shows that once the yield stress is greater than approximately $40 \mathrm{~Pa}$, it is important to consider rheology control for pipeline systems as it is likely that the pipeline flow will be laminar.

Providing consistent rheology material ensures that a system can be designed to operate within controlled parameters, providing benefits to the slurry thickening and preparation processes, the pump and pipeline system, as well as the deposition process. 


\section{ACKNOWLEDGEMENTS}

The technical input and advice from the staff at Paterson and Cooke Consulting Engineers (Pty) Ltd is greatly appreciated.

\section{REFERENCES}

Slatter, P.T. and Wasp, E.J. (2000) The laminar/turbulent transition in large pipes, 10th International Conference on Transport and Sedimentation of Solid Particles, Wroclaw, 4-7 September 2000, ISBN 83-87866-12-1, pp. 389-399. 